\title{
Existence of H-bubbles in a perturbative setting
}

Paolo Caldiroli and Roberta Musina

\begin{abstract}
Given a $C^{1}$ function $H: \mathbb{R}^{3} \rightarrow \mathbb{R}$, we look for $H$-bubbles, i.e., surfaces in $\mathbb{R}^{3}$ parametrized by the sphere $\mathbb{S}^{2}$ with mean curvature $H$ at every regular point. Here we study the case $H(u)=H_{0}(u)+\epsilon H_{1}(u)$ where $H_{0}$ is some "good" curvature (for which there exist $H_{0}$-bubbles with minimal energy, uniformly bounded in $\left.L^{\infty}\right), \epsilon$ is the smallness parameter, and $H_{1}$ is any $C^{1}$ function.
\end{abstract}

\section{Introduction}

In this work we study the existence of $\mathbb{S}^{2}$-type parametric surfaces in the Euclidean space $\mathbb{R}^{3}$, having prescribed mean curvature.

This geometrical problem can be stated in analytical form as follows: given a $C^{1}$ map $H: \mathbb{R}^{3} \rightarrow \mathbb{R}$, find a smooth nonconstant function $\omega: \mathbb{R}^{2} \rightarrow \mathbb{R}^{3}$ satisfying

$$
\left\{\begin{array}{l}
\Delta \omega=2 H(\omega) \omega_{x} \wedge \omega_{y} \quad \text { in } \mathbb{R}^{2} \\
\int_{\mathbb{R}^{2}}|\nabla \omega|^{2}<+\infty
\end{array}\right.
$$

Indeed it is known that such a function $\omega$ turns out to be conformal and if $p=\omega(z)$ is a regular point, then the value $H(p)$ represents the mean curvature of the surface parametrized by $\omega$ at the point $p$. Moreover, denoting by $\sigma: \mathbb{S}^{2} \rightarrow \mathbb{R}^{2}$ the stereographic projection, the mapping $\omega \circ \sigma: \mathbb{S}^{2} \rightarrow \mathbb{R}^{3}$ defines an $\mathbb{S}^{2}$-type parametric surface in $\mathbb{R}^{3}$ having prescribed mean curvature $H$, briefly, an $H$-bubble.

2000 Mathematics Subject Classification: 53A10, 49J10.

Keywords: parametric surfaces, prescribed mean curvature. 
When the prescribed mean curvature is a nonzero constant $H(u) \equiv H_{0}$, Brezis and Coron [2] proved that the only nonconstant solutions to (1.1) are spheres of radius $\left|H_{0}\right|^{-1}$ anywhere placed in $\mathbb{R}^{3}$.

Only recently, the case in which $H$ is nonconstant has been investigated. In particular, in [3] the authors studied the case of a bounded function $H \in$ $C^{1}\left(\mathbb{R}^{3}\right)$ asymptotic to a constant at infinity. Under some global assumptions, the existence of an $H$-bubble having "minimal energy" is proved. This existence result constitutes the starting point of the present work and, for future convenience, let us recall its precise statement.

Firstly, we point out that problem (1.1) has a variational structure. More precisely, $H$-bubbles can be found as critical points of the energy functional

$$
\mathcal{E}_{H}(u)=\frac{1}{2} \int_{\mathbb{R}^{2}}|\nabla u|^{2}+2 \int_{\mathbb{R}^{2}} Q(u) \cdot u_{x} \wedge u_{y},
$$

where $Q: \mathbb{R}^{3} \rightarrow \mathbb{R}^{3}$ is any vectorfield such that $\operatorname{div} Q=H$.

When $H$ is bounded, $\mathcal{E}_{H}$ turns out to be well defined (by continuous extension) and sufficiently regular on some Sobolev space.

Roughly, the integral

$$
\int_{\mathbb{R}^{2}} Q(u) \cdot u_{x} \wedge u_{y}
$$

has the meaning of the algebraic $H$-weighted volume of the region enclosed by range $u$ and it is essentially cubic in $u$. Therefore, the energy functional $\mathcal{E}_{H}$, which is unbounded from below and from above, actually admits a saddle type geometry.

In order to make precise the geometrical structure of $\mathcal{E}_{H}$, we consider the restriction of $\mathcal{E}_{H}$ to the space of smooth functions $C_{c}^{1}\left(\mathbb{R}^{2}, \mathbb{R}^{3}\right)$, and we introduce the value:

$$
c_{H}=\inf _{\substack{u \in C_{c}^{1}\left(\mathbb{R}^{2}, \mathbb{R}^{3}\right) \\ u \neq 0}} \sup _{s>0} \mathcal{E}_{H}(s u)
$$

which represents the mountain pass level along radial paths. In [3], we proved the following existence result.

Theorem 1.1 Let $H \in C^{1}\left(\mathbb{R}^{3}\right)$ be such that

$\left(h_{1}\right) H(u) \rightarrow H_{\infty}$ as $|u| \rightarrow \infty$, for some $H_{\infty} \in \mathbb{R}$,

$\left(h_{2}\right) \sup _{u \in \mathbb{R}^{3}}|\nabla H(u) \cdot u u|<1$,

$\left(h_{3}\right) c_{H}<\frac{4 \pi}{3 H_{\infty}^{2}}$.

Then there exists an $H$-bubble $\omega$ with $\mathcal{E}_{H}(\omega)=c_{H}$. In addition $c_{H}=\inf _{\mathcal{B}_{H}} \mathcal{E}_{H}$ where $\mathcal{B}_{H}$ is the class of the $H$-bubbles. 
We point out that the assumption $\left(h_{2}\right)$ is important in order to get a positive lower bound for the minimal energy of $H$-bubbles. In addition, it is also used to get boundedness (with respect to the Dirichlet norm) of the Palais Smale sequences of $\mathcal{E}_{H}$ and to guarantee that the value $c_{H}$ is an admissible minimax level. The assumption $\left(h_{3}\right)$ is variational in nature and it is verified, for instance, whenever $H>H_{\infty} \geq 0$ on a suitably large set. Moreover, together with $\left(h_{1}\right)$ and $\left(h_{2}\right)$, the hypothesis $\left(h_{3}\right)$ implies that some special Palais Smale sequences of $\mathcal{E}_{H}$ at level $c_{H}$ are bounded even in the strong $L^{\infty}$ topology.

The main difficulty is the lack of compactness, due to the fact that problem (1.1) is invariant with respect to the conformal group. This means that we deal with a problem on the image of $\omega$, rather that on the mapping $\omega$ itself.

If $H$ is constant there is also an invariance with respect to translations on the image. The assumption $\left(h_{3}\right)$ forces $H$ to be nonconstant and allows us to look for minimal $H$-bubbles in some bounded region, recovering some compactness, in a suitable way.

In this paper we investigate the following question: does the existence result stated above persist under perturbation of the prescribed curvature function? More precisely, we consider the case in which

$$
H(u)=H_{0}(u)+\epsilon H_{1}(u):=H_{\epsilon}(u)
$$

where $H_{0} \in C^{1}\left(\mathbb{R}^{3}\right)$ satisfies $\left(h_{1}\right)-\left(h_{3}\right),|\epsilon|$ is small, and $H_{1}: \mathbb{R}^{3} \rightarrow \mathbb{R}$ is any $C^{1}$ function, not necessarily bounded.

Clearly, in general, none of the hypotheses $\left(h_{1}\right)-\left(h_{3}\right)$ is fulfilled by $H_{\epsilon}$. Furthermore, even the corresponding energy functional $\mathcal{E}_{H_{\epsilon}}$ is not well defined on the Sobolev spaces suited to study problem (1.1). Hence no "global" variational approach works, but a localizing argument has to be followed. To this aim, one takes advantage from the fact that the set of minimal $H_{0^{-}}$ bubbles is uniformly bounded in $L^{\infty}$ (thanks to the condition $\left(h_{3}\right)$ ) and then a truncation on the perturbative term $H_{1}$ can be made. The main result of this paper is stated as follows.

Theorem 1.2 Let $H_{0} \in C^{1}\left(\mathbb{R}^{3}\right)$ be such that $\left(h_{1}\right)-\left(h_{3}\right)$ hold, and let $H_{1} \in$ $C^{1}\left(\mathbb{R}^{3}\right)$. Then there is $\bar{\epsilon}>0$ such that for every $\epsilon \in(-\bar{\epsilon}, \bar{\epsilon})$ there exists an $H_{\epsilon}$-bubble $\omega^{\epsilon}$. Furthermore, as $\epsilon \rightarrow 0, \omega^{\epsilon}$ converges (geometrically) to some minimal $H_{0}$-bubble $\omega$. More precisely

$$
\omega^{\epsilon} \circ \sigma \rightarrow \omega \circ \sigma \quad \text { in } C^{1}\left(\mathbb{S}^{2}, \mathbb{R}^{3}\right) .
$$

We remark that the energy of $\omega^{\epsilon}$ is close to the (unperturbed) minimal energy of $H_{0}$-bubbles. However in general we cannot say that $\omega^{\epsilon}$ is a minimal $H_{\epsilon}$-bubble. 
Finally, we remark that Theorem 1.2 cannot be applied in case the unperturbed curvature $H_{0}$ is a constant, since assumption $\left(h_{3}\right)$ is not satisfied. That case is studied in the work [5], with quite different techniques.

The paper is organized as follows. In Section 2 we introduce some notation and the functional setting. We also recall some useful results already discussed in other papers. In Section 3 we state some convergence properties for the energy functional, and finally in Section 4 we give the proof of Theorem 1.2.

\section{Notation and preliminaries}

Denoting by $\sigma: \mathbb{S}^{2} \rightarrow \mathbb{R}^{2}$ the stereographic projection, to every map $u: \mathbb{S}^{2} \rightarrow$ $\mathbb{R}^{3}$ we associate the map $\bar{u}:=u \circ \sigma^{-1}: \mathbb{R}^{2} \rightarrow \mathbb{R}^{3}$. Thus, for example, for $s \geq 1$, the norm of $u$ in $L^{s}\left(\mathbb{S}^{2}, \mathbb{R}^{3}\right)$ is given by

$$
\|u\|_{s}=\left(\int_{\mathbb{R}^{2}}|\bar{u}|^{s} \mu^{2}\right)^{1 / s}
$$

where

$$
\mu(z)=\frac{2}{1+|z|^{2}}, \quad z=(x, y) .
$$

Similarly, if $d u(p): T_{p} \mathbb{S}^{2} \rightarrow \mathbb{R}^{3}$ denotes the gradient of $u$ at $p \in \mathbb{S}^{2}$, and $z=\sigma(p)$, one has

$$
|d u(p)|=|\nabla \bar{u}(z)| \mu(z)^{-1}
$$

where $\nabla$ denotes the standard gradient in $\mathbb{R}^{2}$. Hence, the norm of $|d u|$ in $L^{s}\left(\mathbb{S}^{2}\right)$ is given by

$$
\|d u\|_{s}=\left(\int_{\mathbb{R}^{2}}|\nabla \bar{u}|^{s} \mu^{2-s}\right)^{1 / s} .
$$

In the following, we simply write $H^{1}$ instead of $W^{1,2}\left(\mathbb{S}^{2}, \mathbb{R}^{3}\right)$ and we will often identify any map $u: \mathbb{S}^{2} \rightarrow \mathbb{R}^{3}$ with $\bar{u}:=u \circ \sigma^{-1}: \mathbb{R}^{2} \rightarrow \mathbb{R}^{3}$.

Now, let $H \in C^{1}\left(\mathbb{R}^{3}\right)$ be a given curvature, and let $Q: \mathbb{R}^{3} \rightarrow \mathbb{R}^{3}$ be any smooth vectorfield such that $\operatorname{div} Q=H$. For every $u \in H^{1} \cap L^{\infty}$ let us set

$$
\mathcal{V}_{H}(u)=\int_{\mathbb{R}^{2}} Q(u) \cdot u_{x} \wedge u_{y}
$$

If $u$ is smooth enough, the integral defined by $\mathcal{V}_{H}(u)$ corresponds to the algebraic volume enclosed by the surface parametrized by $u$, with weight $H$, 
and it is independent of the choice of the vectorfield $Q$ (see [9]). Here we choose

$$
Q(u)=m_{H}(u) u, \quad m_{H}(u)=\int_{0}^{1} H(s u) s^{2} d s .
$$

Notice that the following identity holds on $\mathbb{R}^{3}$ :

$$
H(u)=3 m(u)+\nabla m(u) \cdot u .
$$

Denoting by

$$
\mathcal{D}(u)=\frac{1}{2} \int_{\mathbb{R}^{2}}|\nabla u|^{2}
$$

the Dirichlet integral of $u$, the energy functional $\mathcal{E}_{H}: H^{1} \cap L^{\infty} \rightarrow \mathbb{R}$ can be written as:

$$
\mathcal{E}_{H}(u)=\mathcal{D}(u)+2 \mathcal{V}_{H}(u) .
$$

It is proved that if $H$ is bounded, $\mathcal{E}_{H}$ admits a continuous extension on $H^{1}$ (see [9]) and for every $u \in H^{1}$ there exists the directional derivative of $\mathcal{E}_{H}$ at $u$ along any $\varphi \in H^{1} \cap L^{\infty}$ (see [7]), given by

$$
\partial_{\varphi} \mathcal{E}_{H}(u)=\int_{\mathbb{R}^{2}} \nabla u \cdot \nabla \varphi+2 \int_{\mathbb{R}^{2}} H(u) \varphi \cdot u_{x} \wedge u_{y} .
$$

Now, for any $H \in C^{1}\left(\mathbb{R}^{3}\right)$ define

$$
M_{H}=\sup _{u \in \mathbb{R}^{3}}|\nabla H(u) \cdot u u| .
$$

Using (2.3) with $\varphi=u$, and the identity (2.2), one easily obtains the following key estimate

$$
3 \mathcal{E}_{H}(u) \geq\left(1-M_{H}\right) \mathcal{D}(u)+\partial_{u} \mathcal{E}_{H}(u) \text { for } u \in H^{1} \cap L^{\infty} .
$$

Notice that, assuming $M_{H}<1$ (namely, the hypothesis $\left(h_{2}\right)$ of Theorem 1.1), (2.4) immediately implies that if $\left(u^{n}\right) \subset H^{1} \cap L^{\infty}$ is a Palais-Smale sequence for $\mathcal{E}_{H}$ at some level $c \in \mathbb{R}$, then $\sup \left\|\nabla u^{n}\right\|_{2}<+\infty$ and $c \geq 0$.

The condition $M_{H}<1$ enters in an important way also to infer some properties on the mountain pass value $c_{H}$ defined by (1.2). This is stated by the next result, proved in [3].

Lemma 2.1 Let $H \in C^{1}\left(\mathbb{R}^{3}\right)$ satisfy $M_{H}<1$. Then:

(i) If $\omega$ is an $H$-bubble, there holds $\mathcal{E}_{H}(\omega) \geq c_{H}$.

(ii) Given $u \in H^{1} \cap L^{\infty}$, u nonconstant, one has $\sup _{s>0} \mathcal{E}_{H}($ su $)<+\infty$ if and only if there exists $s_{1}>0$ such that $\mathcal{E}_{H}\left(s_{1} u\right)<0$. If this is the case, then $\sup _{s>0} \mathcal{E}_{H}(s u)=\max _{s \in\left[0, s_{1}\right]} \mathcal{E}_{H}(s u)$. 
Remark 2.2 If $H(u) \equiv H_{0} \in \mathbb{R} \backslash\{0\}$ for all $u \in \mathbb{R}^{3}$, then

$$
c_{H_{0}}=\frac{4 \pi}{3 H_{0}^{2}} \text {. }
$$

Indeed, given $u \in C_{c}^{1}\left(\mathbb{R}^{2}, \mathbb{R}^{3}\right), u \neq 0$, the mapping $s \mapsto \mathcal{E}_{H_{0}}(s u)$ admits a critical point $\bar{s}>0$ if and only if $\mathcal{V}_{H_{0}}(u)<0$. In this case

$$
\sup _{s>0} \mathcal{E}_{H_{0}}(s u)=\mathcal{E}_{H_{0}}(\bar{s} u)=\frac{1}{27 H_{0}^{2}} \frac{\mathcal{D}(u)^{3}}{\mathcal{V}_{1}(u)^{2}} .
$$

Then

where

$$
c_{H_{0}}=\frac{S^{3}}{27 H_{0}^{2}}
$$

$$
S=\inf _{\substack{u \in C_{c}^{1}\left(\mathbb{R}^{2}, \mathbb{R}^{3}\right) \\ u \neq 0}} \frac{\mathcal{D}(u)}{\mathcal{V}_{1}(u)^{2 / 3}}
$$

is the classical isoperimetric constant (see [10]). Since $S=\sqrt[3]{36 \pi}$ the conclusion follows.

\section{Some convergence results}

The first convergence result presented in this Section is given by an upper semicontinuity property for the mountain pass level defined by (1.2). This result has been proved in [3].

Lemma 3.1 Let $H \in C^{1}\left(\mathbb{R}^{3}\right)$ satisfy $M_{H}<1$. Let $\left(H_{n}\right) \subset C^{1}\left(\mathbb{R}^{3}\right)$ be a sequence of functions satisfying $M_{H_{n}}<1$, and such that $H_{n} \rightarrow H$ uniformly on compact sets of $\mathbb{R}^{3}$. Then $\lim \sup c_{H_{n}} \leq c_{H}$.

The next result concerns the semicontinuity of the energy functional $\mathcal{E}_{H}$. In general $\mathcal{E}_{H}$ is not lower semicontinuous, because of possible concentration phenomena (see an example by Wente in [10]). However, as stated by the next Lemma, the lower semicontinuity holds true at least along a sequence of solutions.

First, let us introduce the following notation: for every $\rho>0$ set

$$
\bar{M}_{H, \rho}=2 \sup _{|u|<\rho}\left|\left(H(u)-3 m_{H}(u)\right) u\right| .
$$

Notice that $\bar{M}_{H, \rho} \leq M_{H}$. In addition, if $H_{n} \rightarrow H$ uniformly on

$$
\bar{B}_{\rho}=\left\{u \in \mathbb{R}^{3}:|u| \leq \rho\right\},
$$

then $\bar{M}_{H_{n}, \rho} \rightarrow \bar{M}_{H, \rho}$. 
Lemma 3.2 Let $\left(H_{n}\right) \subset C^{1}\left(\mathbb{R}^{3}\right), H \in C^{1}\left(\mathbb{R}^{3}\right)$ and $\rho>0$ be such that:

(i) $H_{n} \rightarrow H$ uniformly on $\bar{B}_{\rho}$,

(ii) $\bar{M}_{H_{n}, \rho} \leq 1$ for every $n \in \mathbb{N}$.

Let $\left(\omega^{n}\right) \subset H^{1} \cap L^{\infty}$ be such that for every $n \in \mathbb{N}$ :

(iii) $\omega^{n}$ is an $H_{n}$-bubble,

(iv) $\left\|\omega^{n}\right\|_{\infty} \leq \rho$,

(v) $\left|\nabla \omega^{n}(0)\right|=\left\|\nabla \omega^{n}\right\|_{\infty}=1$

(vi) $\left\|\nabla \omega^{n}\right\|_{2} \leq C$ for some positive constant $C$.

Then there exists an $H$-bubble $\omega$ such that, for a subsequence, $\omega^{n} \rightarrow \omega$ weakly in $H^{1}$ and strongly in $C_{\text {loc }}^{1}\left(\mathbb{R}^{2}, \mathbb{R}^{3}\right)$. Moreover $\mathcal{E}_{H}(\omega) \leq \liminf \mathcal{E}_{H_{n}}\left(\omega^{n}\right)$.

To prove Lemma 3.2 we will use the following " $\varepsilon$-regularity" Lemma, inspired by a similar result due to Sacks and Uhlenbeck [8] (see also Lemma A.1 in [1]). For its proof, we refer to [3].

Lemma 3.3 Let $H \in C^{1}\left(\mathbb{R}^{3}\right) \cap L^{\infty}$. Then there exist $\varepsilon>0$ such that for every $s>1$ there exists a constant $C_{s}>0$, depending only on $s,\|H\|_{\infty}$, such that if $u \in W_{\text {loc }}^{2, s}\left(D, \mathbb{R}^{3}\right)$ solves $\Delta u=2 H(u) u_{x} \wedge u_{y}$ on an open domain $\Omega \subseteq \mathbb{R}^{2}$, then

$$
\|\nabla u\|_{L^{2}\left(D_{R}(z)\right)} \leq \varepsilon \Rightarrow\|\nabla u\|_{W^{1, s}\left(D_{R / 2}(z)\right)} \leq C_{s} R^{\frac{2}{s}-s}\|\nabla u\|_{L^{2}\left(D_{R}(z)\right)}
$$

for every disc $\overline{D_{R}(z)} \subset \Omega$ with $R \in(0,1)$.

Proof of Lemma 3.2. From the assumption (iv) and (vi), there exists $\omega \in H^{1} \cap L^{\infty}$ such that, for a subsequence, $\omega^{n} \rightarrow \omega$ weakly in $H^{1}$. Now fix an arbitrary $r>0$ and let us prove that $\omega^{n} \rightarrow \omega$ strongly in $C^{1}\left(\bar{D}_{r}, \mathbb{R}^{3}\right)$. By Lemma 3.3, for every $n \in \mathbb{N}$ and for $s>2$ fixed, there exists $\varepsilon_{n}>0$ (which in fact depends only on $\left\|H_{n}\right\|_{L^{\infty}\left(B_{\rho}\right)}$ ) and $C_{s, n}>0$ for which

$$
\left\|\nabla \omega^{n}\right\|_{L^{2}\left(D_{R}(z)\right)} \leq \varepsilon_{n} \Rightarrow\left\|\nabla \omega^{n}\right\|_{H^{1, s}\left(D_{R / 2}(z)\right)} \leq C_{s, n} R^{\frac{2}{s}-2}\left\|\nabla \omega^{n}\right\|_{L^{2}\left(D_{R}(z)\right)}
$$

for every $z \in \mathbb{R}^{2}$ and for every $R \in(0,1)$. By (i), one has $\varepsilon_{n} \geq \varepsilon>0$ and $C_{s, n} \leq C_{s}$ for every $n \in \mathbb{N}$. Since $\left\|\nabla \omega^{n}\right\|_{\infty}=1$, there exists $R \in(0,1)$ and a finite covering $\left\{D_{R / 2}\left(z_{i}\right)\right\}_{i \in I}$ of $\bar{D}_{r}$ such that $\left\|\nabla \omega^{n}\right\|_{L^{2}\left(D_{R}\left(z_{i}\right)\right)} \leq \varepsilon$ for every $n \in \mathbb{N}$ and $i \in I$. Since $\left\|\omega^{n}\right\|_{\infty} \leq \rho$, we have that

$$
\left\|\omega^{n}\right\|_{H^{2, s}\left(D_{R / 2}\left(z_{i}\right)\right)} \leq \bar{C}_{s, \rho}
$$

for some constant $\bar{C}_{s, \rho}>0$ independent of $i \in I$ and $n \in \mathbb{N}$. 
Then the sequence $\left(\omega^{n}\right)$ is bounded in $H^{2, s}\left(D_{r}, \mathbb{R}^{3}\right)$. For $s>2$ the space $H^{2, s}\left(D_{r}, \mathbb{R}^{3}\right)$ is compactly embedded into $C^{1}\left(\bar{D}_{r}, \mathbb{R}^{3}\right)$. Hence $\omega^{n} \rightarrow \omega$ strongly in $C^{1}\left(\bar{D}_{r}, \mathbb{R}^{3}\right)$. By a standard diagonal argument, one concludes that $\omega^{n} \rightarrow \omega$ strongly in $C_{l o c}^{1}\left(\mathbb{R}^{2}, \mathbb{R}^{3}\right)$.

Now we prove that $\omega$ is an $H$-bubble. Indeed, for every $n \in \mathbb{N}$, if $h \in$ $C_{c}^{\infty}\left(\mathbb{R}^{2}, \mathbb{R}^{3}\right)$ then

$$
\int_{\mathbb{R}^{2}} \nabla \omega^{n} \cdot \nabla h+2 \int_{\mathbb{R}^{2}} H_{n}\left(\omega^{n}\right) h \cdot \omega_{x}^{n} \wedge \omega_{y}^{n}=0 .
$$

Since $\omega^{n} \rightarrow \omega$ strongly in $C_{l o c}^{1}\left(\mathbb{R}^{2}, \mathbb{R}^{3}\right)$, passing to the limit, one immediately infers that $\omega$ is a weak solution to (1.1). By regularity theory of $H$-systems (see, e.g., [6]), $\omega$ is a classical, conformal solution to (1.1). In addition $\omega$ is nonconstant, since

$$
|\nabla \omega(0)|=\lim \left|\nabla \omega^{n}(0)\right|=1 .
$$

Hence $\omega$ is an $H$-bubble.

Finally, let us show that

$$
\mathcal{E}_{H}(\omega) \leq \liminf \mathcal{E}_{H_{n}}\left(\omega^{n}\right)
$$

Again by the strong convergence in $C_{l o c}^{1}\left(\mathbb{R}^{2}, \mathbb{R}^{3}\right)$ and by (i) and (iv), for every $R>0$, one has

$$
\mathcal{E}_{H_{n}}\left(\omega^{n}, D_{R}\right) \rightarrow \mathcal{E}_{H}\left(\omega, D_{R}\right)
$$

where we denote

$$
\mathcal{E}_{H_{n}}\left(\omega^{n}, \Omega\right)=\frac{1}{2} \int_{\Omega}\left|\nabla \omega^{n}\right|^{2}+2 \int_{\Omega} m_{H_{n}}\left(\omega^{n}\right) \omega^{n} \cdot \omega_{x}^{n} \wedge \omega_{y}^{n}
$$

(and similarly for $\mathcal{E}_{H}(\omega, \Omega)$ ). Now, fixing $\epsilon>0$, let $R>0$ be such that

$$
\begin{aligned}
\mathcal{E}_{H}\left(\omega, \mathbb{R}^{2} \backslash D_{R}\right) & \leq \epsilon \\
\int_{\mathbb{R}^{2} \backslash D_{R}}|\nabla \omega|^{2} & \leq \epsilon .
\end{aligned}
$$

By (3.2) and (3.1) we have

$$
\begin{aligned}
\mathcal{E}_{H}(\omega) & \leq \mathcal{E}_{H}\left(\omega, D_{R}\right)+\epsilon \\
& =\mathcal{E}_{H_{n}}\left(\omega^{n}, D_{R}\right)+\epsilon+o(1) \\
& =\mathcal{E}_{H_{n}}\left(\omega^{n}\right)-\mathcal{E}_{H_{n}}\left(\omega^{n}, \mathbb{R}^{2} \backslash D_{R}\right)+\epsilon+o(1)
\end{aligned}
$$

with $o(1) \rightarrow 0$ as $n \rightarrow+\infty$. 
Since every $\omega^{n}$ is an $H_{n}$-bubble, using the divergence theorem, for any $R>0$ one has

$$
\begin{aligned}
\frac{1}{2} \int_{\mathbb{R}^{2} \backslash D_{R}}\left|\nabla \omega^{n}\right|^{2}= & 3 \mathcal{E}_{H_{n}}\left(\omega^{n}, \mathbb{R}^{2} \backslash D_{R}\right)-\int_{\partial D_{R}} \omega^{n} \cdot \frac{\partial \omega^{n}}{\partial \nu} \\
& +2 \int_{\mathbb{R}^{2} \backslash D_{R}}\left(H_{n}\left(\omega^{n}\right)-3 m_{H_{n}}\left(\omega^{n}\right)\right) \omega^{n} \cdot \omega_{x}^{n} \wedge \omega_{y}^{n} .
\end{aligned}
$$

Moreover, by definition of $\bar{M}_{H_{n}, \rho}$, we can estimate

$$
2 \int_{\mathbb{R}^{2} \backslash D_{R}}\left(H_{n}\left(\omega^{n}\right)-3 m_{H_{n}}\left(\omega^{n}\right)\right) \omega^{n} \cdot \omega_{x}^{n} \wedge \omega_{y}^{n} \leq \frac{\bar{M}_{H_{n}, \rho}}{2} \int_{\mathbb{R}^{2} \backslash D_{R}}\left|\nabla \omega^{n}\right|^{2} .
$$

Thus, we obtain

$$
\begin{aligned}
-\mathcal{E}_{H_{n}}\left(\omega^{n}, \mathbb{R}^{2} \backslash D_{R}\right) & \leq-\frac{1}{3} \int_{\partial D_{R}} \omega^{n} \cdot \frac{\partial \omega^{n}}{\partial \nu}-\frac{1-\bar{M}_{H_{n}, \rho}}{6} \int_{\mathbb{R}^{2} \backslash D_{R}}\left|\nabla \omega^{n}\right|^{2} \\
& \leq-\frac{1}{3} \int_{\partial D_{R}} \omega^{n} \cdot \frac{\partial \omega^{n}}{\partial \nu}
\end{aligned}
$$

because of the assumption (ii). Using again the $C_{l o c}^{1}$ convergence of $\omega^{n}$ to $\omega$, as well as the fact that $\omega$ is an $H$-bubble, we obtain that

$$
\begin{aligned}
\lim _{n \rightarrow+\infty}\left|\int_{\partial D_{R}} \omega^{n} \cdot \frac{\partial \omega^{n}}{\partial \nu}\right| & =\left|\int_{\partial D_{R}} \omega \cdot \frac{\partial \omega}{\partial \nu}\right| \\
& =\left|\int_{\mathbb{R}^{2} \backslash D_{R}}\left(\omega \cdot \Delta \omega+|\nabla \omega|^{2}\right)\right| \\
& =\left|\int_{\mathbb{R}^{2} \backslash D_{R}}\left(2 H(\omega) \omega \cdot \omega_{x} \wedge \omega_{y}+|\nabla \omega|^{2}\right)\right| \\
& \leq\left(\|\omega\|_{\infty}\|H\|_{\infty}+1\right) \int_{\mathbb{R}^{2} \backslash D_{R}}|\nabla \omega|^{2} \\
& \leq\left(\|\omega\|_{\infty}\|H\|_{\infty}+1\right) \epsilon
\end{aligned}
$$

thanks to (3.3). Finally, (3.4), (3.6) and (3.7) imply

$$
\mathcal{E}_{H}(\omega) \leq \mathcal{E}_{H_{n}}\left(\omega^{n}\right)+C \epsilon+o(1)
$$

for some positive constant $C$ independent of $\epsilon$ and $n$. Hence, the conclusion follows. 
As a consequence of Lemma 3.2, one obtains the following result.

Lemma 3.4 Let $H \in C^{1}\left(\mathbb{R}^{3}\right)$ satisfy $\left(h_{1}\right)-\left(h_{3}\right)$. Then there exists $\rho>0$ (depending on $\|H\|_{\infty}$, on $\mu_{H}:=1-M_{H}>0$ and on $\delta_{H}:=\frac{4 \pi}{3 H_{\infty}^{2}}-c_{H}>0$ ) such that

$$
\|\omega\|_{\infty} \leq \rho
$$

for every $H$-bubble $\omega$ with $\mathcal{E}_{H}(\omega)=c_{H}$.

Proof. First, notice that $\omega$ is smooth by the regularity theory for $H$-systems (see for example [6]). In addition, if $\mathcal{E}_{H}(\omega)=c_{H}$, then by (2.4), we get

$$
\int_{\mathbb{R}^{2}}|\nabla \omega|^{2} \leq \frac{6 c_{H}}{1-M_{H}}
$$

Moreover, by a corollary to a Grüter's estimate [6] (see [3, proof of Theorem 1.1]), one has

$$
\operatorname{diam} \omega \leq C\left(1+\int_{\mathbb{R}^{2}}|\nabla \omega|^{2}\right)
$$

where

$$
\operatorname{diam} \omega=\sup _{z, z^{\prime} \in \mathbb{R}^{2}}\left|\omega(z)-\omega\left(z^{\prime}\right)\right|,
$$

and $C$ is a positive constant depending only on $\|H\|_{\infty}$. Hence

$$
\operatorname{diam} \omega \leq \frac{C\left(\|H\|_{\infty}\right)}{1-M_{H}}=: \rho .
$$

To conclude, it is enough to show that there exists $\rho_{0}>0$ such that $|\omega(0)| \leq \rho_{0}$, for every $H$-bubble $\omega$ with $\mathcal{E}_{H}(\omega)=c_{H}$. Arguing by contradiction, suppose that $\left|\omega^{n}(0)\right| \rightarrow+\infty$ for a sequence $\left(\omega^{n}\right)$ of $H$-bubbles with $\mathcal{E}_{H}\left(\omega^{n}\right)=c_{H}$. Set

$$
\bar{\omega}^{n}=\omega^{n}-\omega^{n}(0) \quad \text { and } \quad H_{n}(u)=H\left(u+\omega^{n}(0)\right) .
$$

Then $H_{n} \rightarrow H_{\infty}$ uniformly on compact sets. This also implies that $\bar{M}_{H_{n}, \rho} \rightarrow 0$ as $n \rightarrow+\infty$. Moreover, thanks to the conformal invariance, we may suppose $\left|\nabla \bar{\omega}^{n}(0)\right|=\left\|\nabla \bar{\omega}^{n}\right\|_{\infty}=1$. Clearly, for every $n \in \mathbb{N}, \bar{\omega}^{n}$ is an $H_{n}$-bubble. In addition, (3.8) and (3.9) imply that

$$
\sup \left(\left\|\bar{\omega}^{n}\right\|_{\infty}+\left\|\nabla \bar{\omega}^{n}\right\|_{2}\right)<+\infty .
$$

Hence we are in position to apply Lemma 3.2, obtaining that

$$
\liminf \mathcal{E}_{H_{n}}\left(\bar{\omega}^{n}\right) \geq \mathcal{E}_{H_{\infty}}(\bar{\omega})
$$

where $\bar{\omega}$ is some $H_{\infty}$-bubble. But $\mathcal{E}_{H_{n}}\left(\bar{\omega}^{n}\right)=\mathcal{E}_{H}\left(\omega^{n}\right)=c_{H}$, and, by Lemma 2.1, $\mathcal{E}_{H_{\infty}}(\bar{\omega}) \geq c_{H_{\infty}}$. Hence, thanks to Remark 2.2, we obtain $c_{H} \geq \frac{4 \pi}{3 H_{\infty}^{2}}$, in contradiction with $\left(h_{3}\right)$. This concludes the proof. 
Finally we state a sufficient condition in order to have strong convergence in $H^{1}$ along a sequence of $H_{n}$-bubbles, when $H_{n}$ converges to some limit curvature $H$.

Lemma 3.5 Let $\left(H_{n}\right) \subset C^{1}\left(\mathbb{R}^{3}\right), H \in C^{1}\left(\mathbb{R}^{3}\right)$ and $\rho>0$ be such that:

(i) $H_{n} \rightarrow H$ uniformly on $\bar{B}_{\rho}$,

(ii) $\bar{M}_{H, \rho}<1$.

Let $\left(\omega^{n}\right) \subset H^{1} \cap L^{\infty}$ and $\omega \in H^{1} \cap L^{\infty}$ be such that:

(iii) $\omega$ is an $H$-bubble and $\omega^{n}$ is an $H_{n}$-bubble for every $n \in \mathbb{N}$,

(iv) $\left\|\omega^{n}\right\|_{\infty} \leq \rho$ for every $n \in \mathbb{N}$,

$(\mathrm{v}) \omega^{n} \rightarrow \omega$ weakly in $H^{1}$ and strongly in $C_{l o c}^{1}\left(\mathbb{R}^{2}, \mathbb{R}^{3}\right)$,

(vi) $\mathcal{E}_{H_{n}}\left(\omega^{n}\right) \rightarrow \mathcal{E}_{H}(\omega)$.

Then $\omega^{n} \rightarrow \omega$ strongly in $H^{1}$.

Proof. First, notice that assumption (v) guarantees that

$$
\nabla \omega^{n} \rightarrow \nabla \omega \quad \text { in } L_{l o c}^{2} .
$$

Therefore, in order to have strong convergence of the gradients in $L^{2}$ it suffices to prove that

$$
\forall \epsilon>0, \exists R>0 \text { such that } \limsup \int_{\mathbb{R}^{2} \backslash D_{R}}\left|\nabla \omega^{n}\right|^{2} \leq \epsilon .
$$

Furthermore, assumption (iv), together with Rellich theorem, will readily lead to the conclusion. In order to prove (3.10), we will use some estimates already seen in the proof of Lemma 3.2.

In particular, fixing an arbitrary $\varepsilon>0$ and $R>0$ according to (3.2) and (3.3), by (3.4), (3.5), and (3.7), we have

$$
\frac{1-\bar{M}_{H_{n}, \rho}}{6} \int_{\mathbb{R}^{2} \backslash D_{R}}\left|\nabla \omega^{n}\right|^{2} \leq \mathcal{E}_{H_{n}}\left(\omega^{n}\right)-\mathcal{E}_{H}(\omega)+C \varepsilon+o(1) .
$$

Thanks to (i), for $n \in \mathbb{N}$ large one has $\bar{M}_{H_{n}, \rho} \leq \bar{M}$ for some $\bar{M}<1$. Moreover $\mathcal{E}_{H_{n}}\left(\omega^{n}\right) \rightarrow \mathcal{E}_{H}(\omega)$, by hypothesis, and then we obtain

$$
\frac{1-\bar{M}}{6} \int_{\mathbb{R}^{2} \backslash D_{R}}\left|\nabla \omega^{n}\right|^{2} \leq C \varepsilon+o(1) .
$$

that, up to an insignificant multiplicative constant (independent of $\epsilon$ ), is the desired estimate (3.10), since $\bar{M}<1$. 
Finally, we show that strong convergence in $H^{1}$ of a sequence of $H_{n}$-bubbles implies convergence in $C^{1}\left(\mathbb{S}^{2}, \mathbb{R}^{3}\right)$.

Lemma 3.6 Let $\left(H_{n}\right) \subset C^{1}\left(\mathbb{R}^{3}\right), H \in C^{1}\left(\mathbb{R}^{3}\right)$ and $\rho>0$ be such that $H_{n} \rightarrow H$ uniformly on $\bar{B}_{\rho}$. Let $\left(\omega^{n}\right) \subset H^{1} \cap L^{\infty}$ and $\omega \in H^{1} \cap L^{\infty}$ be such that:

(i) $\omega$ is an $H$-bubble and $\omega^{n}$ is an $H_{n}$-bubble for every $n \in \mathbb{N}$,

(ii) $\left\|\omega^{n}\right\|_{\infty} \leq \rho$ for every $n \in \mathbb{N}$,

(iii) $\omega^{n} \rightarrow \omega$ strongly in $H^{1}$.

Then $\omega^{n} \circ \sigma \rightarrow \omega \circ \sigma$ in $C^{1}\left(\mathbb{S}^{2}, \mathbb{R}^{3}\right)$.

Proof. Setting $\hat{\omega}^{n}(z)=\omega^{n}\left(z^{-1}\right)$ (in complex notation), one easily checks that $\hat{\omega}^{n}$ is an $H_{n}$-bubble. Similarly, $\hat{\omega}(z)=\omega\left(z^{-1}\right)$ is an $H$-bubble. Let $\varepsilon>0$ be given according to Lemma 3.3 and let $R>2$ be such that

$$
\int_{\mathbb{R}^{2} \backslash D_{1 / R}}|\nabla \omega|^{2}<\varepsilon .
$$

Since $\omega^{n} \rightarrow \omega$ strongly in $H^{1}$, for $n \in \mathbb{N}$ large enough one has

$$
\int_{D_{R}}\left|\nabla \hat{\omega}^{n}\right|^{2}=\int_{\mathbb{R}^{2} \backslash D_{1 / R}}\left|\nabla \omega^{n}\right|^{2}<\varepsilon .
$$

Hence, by Lemma 3.3, one obtains

$$
\left\|\nabla \hat{\omega}^{n}\right\|_{W^{1, s}\left(D_{R / 2}\right)} \leq C_{s} R^{\frac{2}{s}-s}\left\|\nabla \hat{\omega}^{n}\right\|_{L^{2}\left(D_{R}\right)} \leq C
$$

for every $n \in \mathbb{N}$ large enough. Since for $s>2 W^{1, s}\left(D_{R / 2}\right)$ is compactly embedded into $C^{0}\left(D_{R / 2}\right)$, we obtain

$$
\hat{\omega}^{n} \rightarrow \hat{\omega} \text { in } C^{1}\left(D_{R / 2}\right) .
$$

Now, let $z_{1}, \ldots, z_{k}$ and $r \in(0,1)$ be such that $\overline{D_{2 / R}} \subset D_{r}\left(z_{1}\right) \cup \ldots \cup D_{r}\left(z_{k}\right)$ and $\int_{D_{2 r}\left(z_{j}\right)}|\nabla \omega|^{2}<\varepsilon$ for every $j=1, \ldots, k$. Applying again Lemma 3.3, we infer that for every $j=1, \ldots, k$ one has $\left\|\nabla \omega^{n}\right\|_{W^{1, s}\left(D_{r}\left(z_{j}\right)\right)} \leq C$ and then $\omega^{n} \rightarrow \omega$ in $C^{1}\left(D_{r}\left(z_{j}\right)\right)$. Hence

$$
\omega^{n} \rightarrow \omega \text { in } C^{1}\left(D_{2 / R}\right) .
$$

Since $\left|\nabla \hat{\omega}^{n}(z)\right|=|z|^{-2}\left|\nabla \omega^{n}\left(z^{-1}\right)\right|$, (3.11) and (3.12) imply

$$
\begin{aligned}
& \sup _{z \in \mathbb{R}^{2}}\left|\omega^{n}(z)-\omega(z)\right| \rightarrow 0 \\
& \sup _{z \in \mathbb{R}^{2}}\left|\nabla\left(\omega^{n}-\omega\right)(z) \mu(z)^{-1}\right| \rightarrow 0 .
\end{aligned}
$$

Then, by (2.1), the thesis follows. 


\section{Proof of Theorem 1.2}

Let $H_{0} \in C^{1}\left(\mathbb{R}^{3}\right)$ satisfy $\left(h_{1}\right)-\left(h_{3}\right)$ and let $R_{0}>0$ be such that $\|\omega\|_{\infty} \leq R_{0}$ for every minimal $H_{0}$-bubble $\omega$, according to Lemma 3.4.

Fixing $H_{1} \in C^{1}\left(\mathbb{R}^{3}\right)$, for every $\epsilon \in(-1,1)$ let

$$
H_{\epsilon}=H_{0}+\epsilon H_{1} \text {. }
$$

Now, let $\tilde{H}_{1}: \mathbb{R}^{3} \rightarrow \mathbb{R}$ of class $C^{1}$ with $\left\|\tilde{H}_{1}\right\|_{\infty}<+\infty,\left\|\nabla \tilde{H}_{1}\right\|_{\infty}<+\infty$, and $\tilde{H}_{1}(u)=H_{1}(u)$ as $|u| \leq \tilde{R}$, for some $\tilde{R}>R_{0}$.

Moreover, let $0<r_{\epsilon}<R_{\epsilon}$ and let $\chi_{\epsilon}: \mathbb{R}^{3} \rightarrow \mathbb{R}$ be a $C^{1}$, radial, cut off function such that $\chi_{\epsilon}(u)=1$ as $|u| \leq r_{\epsilon}, \chi_{\epsilon}(u)=0$ as $|u| \geq R_{\epsilon}$, and $\left|\nabla \chi_{\epsilon}(u)\right| \leq \frac{2}{R_{\epsilon}-r_{\epsilon}}$ for all $u$. Here $r_{\epsilon}$ and $R_{\epsilon}$ are asked to satisfy the following conditions:

$$
\begin{aligned}
& \tilde{R}<r_{\epsilon} \text { for every }|\epsilon|<1, \\
& R_{\epsilon}-r_{\epsilon} \geq 1 \text { for every }|\epsilon|<1, \\
& |\epsilon| R_{\epsilon}^{2} \rightarrow 0 \text { as } \epsilon \rightarrow 0 \\
& r_{\epsilon} \rightarrow+\infty \text { as } \epsilon \rightarrow 0 .
\end{aligned}
$$

Finally, for $|\epsilon|<1$ let

$$
\tilde{H}_{\epsilon}=H_{0}+\epsilon \chi_{\epsilon} \tilde{H}_{1}
$$

Our strategy is to show firstly that for $|\epsilon|$ small there exists a minimal $\tilde{H}_{\epsilon}$-bubble. Secondly, we will prove that if $\omega^{\epsilon}$ is a minimal $\tilde{H}_{\epsilon^{-}}$bubble with $\left|\nabla \omega^{\epsilon}(0)\right|=\left\|\nabla \omega^{\epsilon}\right\|_{\infty}=1$, then for every sequence $\epsilon_{n} \rightarrow 0$ there exists a minimal $H_{0}$-bubble $\omega$ such that, for a subsequence, $\omega^{\epsilon_{n}} \circ \sigma \rightarrow \omega \circ \sigma$ in $C^{1}\left(\mathbb{S}^{2}, \mathbb{R}^{3}\right)$. In particular, this will imply that any minimal $\tilde{H}_{\epsilon}$-bubble stays in the region $|u|<\tilde{R}$, and thus it is an $H_{\epsilon}$-bubble.

Lemma 4.1 There exists $\epsilon_{0}>0$ (depending on $\left\|\tilde{H}_{1}\right\|_{\infty}$ and on $\left\|\nabla \tilde{H}_{1}\right\|_{\infty}$ ) such that for every $|\epsilon|<\epsilon_{0}$ :

(i) $\tilde{H}_{\epsilon}(u) \rightarrow H_{\infty}$ as $|u| \rightarrow+\infty$,

(ii) $\sup _{u \in \mathbb{R}^{3}}\left|\nabla \tilde{H}_{\epsilon}(u) \cdot u u\right|:=M_{\tilde{H}_{\epsilon}} \leq \bar{M}<1$,

(iii) $c_{\tilde{H}_{\epsilon}} \leq \frac{4 \pi}{3 H_{\infty}^{2}}-\delta_{0}$, for some $\delta_{0}>0$ independent of $\epsilon$.

Proof. Part (i) follows by the fact that $\tilde{H}_{\epsilon}(u)=H_{0}(u)$ as $|u| \geq R_{\epsilon}$. For the same reason, $\left|\nabla \tilde{H}_{\epsilon}(u) \cdot u u\right|=\left|\nabla H_{0}(u) \cdot u u\right| \leq M_{H_{0}}<1$ as $|u| \geq R_{\epsilon}$. For $|u|<R_{\epsilon}$ direct computations easily give

$$
\left|\nabla \tilde{H}_{\epsilon}(u) \cdot u u\right| \leq M_{H_{0}}+|\epsilon| R_{\epsilon}^{2}\left\|\nabla \tilde{H}_{1}\right\|_{\infty}+\frac{2|\epsilon| R_{\epsilon}^{2}}{R_{\epsilon}-r_{\epsilon}}\left\|\tilde{H}_{1}\right\|_{\infty} .
$$


Hence (ii) follows, thanks to (4.2)-(4.3). Finally, let us check (iii). By hypothesis, there exists $u \in C_{c}^{1}\left(D, \mathbb{R}^{3}\right), u \neq 0$, such that

$$
\sup _{s>0} \mathcal{E}_{H_{0}}(s u) \leq \frac{4 \pi}{3 H_{\infty}^{2}}-2 \delta_{0}
$$

for some $\delta_{0}>0$. By Lemma 2.1, part (i),

$$
\sup _{s>0} \mathcal{E}_{H_{0}}(s u)=\max _{s \in\left[0, s_{1}\right]} \mathcal{E}_{H_{0}}(s u) \quad \text { for some } s_{1}>0 .
$$

Setting $\rho_{1}=s_{1}\|u\|_{\infty}$, by (4.4), $r_{\epsilon} \geq \rho_{1}$ for $|\epsilon|$ small, and then,

$$
\mathcal{E}_{\tilde{H}_{\epsilon}}(s u)=\mathcal{E}_{H_{0}}(s u)+\epsilon \mathcal{V}_{\tilde{H}_{1}}(s u) \text { for } s \in\left[0, s_{1}\right] \text {. }
$$

In particular, $\mathcal{E}_{\tilde{H}_{\epsilon}}\left(s_{1} u\right)=\mathcal{E}_{H_{0}}\left(s_{1} u\right)+o(1)$ with $o(1) \rightarrow 0$ as $\epsilon \rightarrow 0$. Hence, for $|\epsilon|$ small, $\mathcal{E}_{\tilde{H}_{\epsilon}}\left(s_{1} u\right)<0$ and, using again Lemma 2.1, part (i),

$$
\sup _{s>0} \mathcal{E}_{\tilde{H}_{\epsilon}}(s u)=\max _{s \in\left[0, s_{1}\right]} \mathcal{E}_{\tilde{H}_{\epsilon}}(s u) .
$$

Finally, (4.5) yields

$$
\max _{s \in\left[0, s_{1}\right]} \mathcal{E}_{\tilde{H}_{\epsilon}}(s u)=\max _{s \in\left[0, s_{1}\right]} \mathcal{E}_{H_{0}}(s u)+o(1) .
$$

In conclusion, for $|\epsilon|$ small enough, one obtains

$$
\sup _{s>0} \mathcal{E}_{\tilde{H}_{\epsilon}}(s u) \leq \frac{4 \pi}{3 H_{\infty}^{2}}-\delta_{0}
$$

namely, (iii).

In the next step we state the existence of minimal $\tilde{H}_{\epsilon}$-bubbles satisfying some uniform estimates.

Lemma 4.2 There exists $\epsilon_{0}>0$ such that for every $|\epsilon|<\epsilon_{0}$ there is a $\tilde{H}_{\epsilon}$-bubble $\omega^{\epsilon}$ satisfying:

(i) $\mathcal{E}_{\tilde{H}_{\epsilon}}\left(\omega^{\epsilon}\right)=c_{\tilde{H}_{\epsilon}}$,

(ii) $\left\|\nabla \omega^{\epsilon}\right\|_{2} \leq C$,

(iii) $\left\|\omega^{\epsilon}\right\|_{\infty} \leq C$,

where $C$ is a positive constant independent of $\epsilon$.

Proof. The existence of $\tilde{H}_{\epsilon}$-bubbles $\omega^{\epsilon}$ with $\mathcal{E}_{\tilde{H}_{\epsilon}}\left(\omega^{\epsilon}\right)=c_{\tilde{H}_{\epsilon}}$, for $|\epsilon|$ small, is guaranteed by Lemma 4.1 and by Theorem 1.1. The estimate (ii) follows by (2.4), by (i) and by Lemma 4.1, parts (ii) and (iii). Finally (iii) can be deduced by Lemma 3.4, noticing that $M_{\tilde{H}_{\epsilon}} \leq \bar{M}<1$ and $c_{\tilde{H}_{\epsilon}} \leq \frac{4 \pi}{3 H_{\infty}^{2}}-\delta_{0}$ with $\delta_{0}>0$. 
For $|\epsilon|$ small, let $\omega^{\epsilon}$ be the $\tilde{H}_{\epsilon}$-bubble given by Lemma 4.2 . Note that, since $r_{\epsilon} \rightarrow+\infty$, whereas $\omega^{\epsilon}$ are uniformly bounded in $L^{\infty}$, for $|\epsilon|$ small $\omega^{\epsilon}$ is in fact an $\left(H_{0}+\epsilon \tilde{H}_{1}\right)$-bubble. Actually, we need a sharper estimate on the $L^{\infty}$ norms of $\omega^{\epsilon}$, and precisely $\left\|\omega^{\epsilon}\right\|_{\infty} \leq \tilde{R}$, so that we can conclude that $\omega^{\epsilon}$ are $H_{\epsilon}$-bubbles. This is the last step, and it will be accomplished in the sequel.

Notice that, by the invariance of problem (1.1) with respect to dilation, translation and inversion, we may suppose that

$$
\left\|\nabla \omega^{\epsilon}\right\|_{\infty}=\left|\nabla \omega^{\epsilon}(0)\right|=1
$$

By the uniform bounds (ii) and (iii) stated in Lemma 4.2, using Lemma 3.2, and reminding that $\tilde{H}_{\epsilon} \rightarrow H_{0}$ uniformly on compact sets, we may also suppose that there exists an $H_{0}$-bubble $\omega$ such that (for a subsequence) $\omega^{\epsilon} \rightarrow \omega$ weakly in $H^{1}$ and in $C_{l o c}^{1}\left(\mathbb{R}^{2}, \mathbb{R}^{3}\right)$. Moreover

$$
\mathcal{E}_{H_{0}}(\omega) \leq \liminf \mathcal{E}_{\tilde{H}_{\epsilon}}\left(\omega^{\epsilon}\right) \text {. }
$$

Lemma $4.3 \omega^{\epsilon} \circ \sigma \rightarrow \omega \circ \sigma$ in $C^{1}\left(\mathbb{S}^{2}, \mathbb{R}^{3}\right)$.

Proof. Notice that $\mathcal{E}_{\tilde{H}_{\epsilon}}\left(\omega^{\epsilon}\right)=c_{\tilde{H}_{\epsilon}}$ and, since $M_{\tilde{H}_{\epsilon}}<1$, by Lemma 3.1, $\lim \sup c_{\tilde{H}_{\epsilon}} \leq c_{H_{0}}$. Hence, by (4.7) and by Lemma 2.1 we infer that $\omega$ is a minimal $H_{0}$-bubble, namely $\mathcal{E}_{H_{0}}(\omega)=c_{H_{0}}$, and

$$
\mathcal{E}_{H_{0}}(\omega)=\lim \mathcal{E}_{\tilde{H}_{\epsilon}}\left(\omega^{\epsilon}\right) \text {. }
$$

Hence all the hypotheses stated in Lemma 3.5 are fulfilled and thus we conclude that $\omega^{\epsilon} \rightarrow \omega$ strongly in $H^{1}$. Then we apply Lemma 3.6 to obtain the thesis.

Thus, in particular, $\omega^{\epsilon} \rightarrow \omega$ uniformly on $\mathbb{R}^{2}$, and, since $\omega$ is a minimal $H_{0}$-bubble, $\|\omega\|_{\infty} \leq R_{0}$ and $\left\|\omega^{\epsilon}\right\|_{\infty}<\tilde{R}$ for $|\epsilon|$ small enough. Hence $\omega^{\epsilon}$ is an $H_{\epsilon}$-bubble and Lemma 4.3 completes the proof of Theorem 1.2.

\section{References}

[1] Bethuel, F. And Rey, O.: Multiple solutions to the Plateau problem for nonconstant mean curvature. Duke Math. J. 73 (1994), no. 3, 593-646.

[2] Brezis, H. And Coron, J. M.: Convergence of solutions of H-systems or how to blow bubbles. Arch. Rational Mech. Anal. 89 (1985), no. 1, 21-56.

[3] Caldiroli, P. and Musina, R.: Existence of minimal H-bubbles. Commun. Contemp. Math. 4 (2002), no. 2, 177-210. 
[4] Caldiroli, P. and Musina, R.: $\mathbb{S}^{2}$-type parametric surfaces with prescribed mean curvature and minimal energy. In Nonlinear equations: methods, models and applications (Bergamo, 2001), 61-77. Progr. Nonlinear Differential Equations Appl. 54. Birkhäuser, Basel, 2003.

[5] Caldiroli, P. and Musina, R.: $H$-bubbles in a perturbative setting: the finite-dimensional reduction method. Duke Math. J. 122 (2004), 457-485.

[6] Grüter, M.: Regularity of weak H-surfaces. J. Reine Angew. Math. 329 (1981), 1-15.

[7] Hildebrandt, S. and Kaul, H.: Two-dimensional variational problems with obstructions, and Plateau's problem for H-surfaces in a Riemannian manifold. Comm. Pure Appl. Math. 25 (1972), 187-223.

[8] Sacks, J. And Uhlenbeck, K.: The existence of minimal immersions of 2-spheres. Ann. of Math. (2) 113 (1981), no. 1, 1-24.

[9] Steffen, K.: Isoperimetric inequalities and the problem of Plateau. Math. Ann. 222 (1976), no. 2, 97-144.

[10] Wente, H.: An existence theorem for surfaces of constant mean curvature. J. Math. Anal. Appl. 26 (1969), 318-344.

Recibido: 28 de octubre de 2002

Paolo Caldiroli

Dipartimento di Matematica

Università di Torino

via Carlo Alberto, 10

10123 Torino, Italy

caldiroli@dm.unito.it

Roberta Musina

Dipartimento di Matematica ed Informatica

Università di Udine

via delle Scienze, 206

33100 Udine, Italy

musina@dimi.uniud.it

Work supported by M.U.R.S.T. progetto di ricerca "Metodi Variazionali ed Equazioni Differenziali Nonlineari" (cofin. 2001/2002). 